\title{
A Simulation Model for Nakagami-m Fading Channels, $m<1$
}

\author{
Kun-Wah Yip, Member, IEEE, and Tung-Sang Ng, Senior Member, IEEE
}

\begin{abstract}
This paper proposes a mathematical model for simulating a Nakagami- $m$ fading channel, $m<1$. It is shown that the $m$ fading process can be expressed as a product of a square-root-beta process and a complex Gaussian process. Extension of the proposed model to simulate correlated diversity channels is also given. $\mathrm{Nu}-$ merical results demonstrate that statistical properties of the samples generated from the proposed model are close to the required ones.
\end{abstract}

Index Terms-Fading channels, HF channels, simulation, stochastic processes.

\section{INTRODUCTION}

$\mathbf{R}$ AYLEIGH distribution has been shown to be appropriate for modeling the short-term fading statistics of a high-frequency (HF) ionospheric channel [1]. However, it is possible that the fading becomes more severe than Rayleigh fading as a result of high variability of the HF channel. The phenomenon of fading more severe than Rayleigh fading was observed by Nakagami [2] in a series of channel measurements for some long-distance HF communication links. His measurement results indicate that the $m$ distribution with the fading parameter $m$ in the range $0.5 \leq m<1$ is useful for modeling the fading characteristic of an HF channel when the fading is more severe than Rayleigh fading. Note that the Rayleigh distribution is a special case of the $m$ distribution when $m=1$.

In the design of an HF communication link, the system designer may want to ensure that the performance of the communication link is satisfactory not only in a Rayleigh fading environment but also in an environment characterized by fading more severe than Rayleigh fading. The need for a robust communication link arises when reliability is a concern, such as in tactical command, control, and communication systems. To perform computer or hardware simulation of an HF communication link operating over an $m$ fading channel for $m<1$, one is required to generate a complex random process that fits a given Doppler power spectrum where the amplitude follows an $m$ distribution with $m<1$. Although there are a number of known techniques and simulation systems that can be used to generate a complex Gaussian process for simulating a Rayleigh fading

Paper approved by N. C. Beaulieu, the Editor for Wireless Communication Theory of the IEEE Communications Society. Manuscript received May 4, 1998; revised December 30, 1998 and June 7, 1999. This work was supported in part by the Hong Kong Research Grants Council, the University Research Committee, and the CRCG of The University of Hong Kong. This paper was presented in part at GLOBECOM, Sydney, Australia, November 8-12, 1998.

The authors are with the Department of Electrical and Electronic Engineering, The University of Hong Kong, Hong Kong (e-mail: kwyip@hkueee.hku.hk; tsng@ @kueee.hku.hk).

Publisher Item Identifier S 0090-6778(00)01582-8. channel (e.g., [3]-[8]), techniques for simulating an $m$ fading channel for $m<1$ are relatively few [8], [9].

In Section II-A, we propose a mathematical model that enables one to generate a complex random process for simulating an $m$ fading channel, $m<1$. Emphasis is given to applications of the proposed model for HF communications. Since the original $m$ fading channel model [2] does not provide a phase characterization, we assume that the channel phase shift is uniformly distributed over $[0,2 \pi)$. This assumption seems reasonable for an $m$ fading channel where the channel fading is more severe than Rayleigh fading. It is shown that the complex random process used for simulating an $m$ fading channel, $m<$ 1 , can be expressed as a product of a complex Gaussian process and a square-root-beta process. The square-root-beta process can be realized by a nonlinear transformation of a Gaussian process. Since a number of computationally efficient techniques have been developed for generating Gaussian processes, an advantage of using the proposed model for simulation is that these efficient techniques can be utilized. In Section II-B, we extend the proposed model for simulation of correlated diversity channels.

Advantages of the proposed model over earlier ones are detailed as follows. Pätzold et al. [8] have developed a method for realizing a general $m$ fading process by using Rice's sum of sinusoids. However, selection of amplitude values for sinusoids is rather complex except in the special case of $m=1$, that is, if the channel is a Rayleigh fading channel. In this special case, the simulated waveform is a complex Gaussian process and amplitude values are easily determined. Using the proposed model for simulating an $m$ fading channel, $m<1$, one can avoid the difficult task of determining amplitude values for sinusoids. Furthermore, the proposed model requires the generation of a complex Gaussian process; simulation using the proposed model can therefore take advantage of the efficient technique developed in [8] for the generation of this process. In [9], a first-order hidden Markov model is used to model a Nakagami- $m$ fading process. Although not indicated in [9], this model can be used to generate a fading process that simulates the behavior of the amplitude, say $r(t)$, of an $m$ fading channel. After generating another random process that simulates the phase, say $\theta(t)$, one may generate a complex random process $z(t)=r(t) e^{j \theta(t)}$ that simulates an $m$ fading channel. The difficulty in the implementation of this approach is to determine autocorrelation functions of $r(t)$ and $\theta(t)$ based on a knowledge of the autocorrelation function of $z(t)$, which is usually given in practice. The proposed model bypasses generating $r(t)$ and $\theta(t)$ separately, so that the difficult task of determining their autocorrelation functions is not required. We should also mention that based on the theoretical 
work of Braun and Dersch [10], Dersch and Rüegg [11] have developed a model for simulating Nakagami- $m$ fading channels for urban mobile communications. The model is, however, limited only for the case $m \geq 1$.

The rest of the paper is organized as follows. In Section III, details on the implementation of the proposed simulation model are given. Numerical examples illustrating the use of the proposed model follow in Section IV. Finally, conclusions are drawn in Section V.

\section{Simulation MODEL}

\section{A. Theoretical Development}

Let $z(t)=r(t) e^{j \theta(t)}$ be a wide-sense stationary (WSS) complex random process that is characterized by the autocorrelation function

$$
R_{z}(\Delta t)=E\left\{z(t) z^{*}(t+\Delta t)\right\}
$$

and satisfies the following properties.

1) $r(t)$ is Nakagami- $m$ distributed with the second moment $\Omega=R_{z}(0)$ and the fading parameter $m=\Omega^{2} / E\left\{\left(r(t)^{2}-\Omega\right)^{2}\right\}$ limited in the range $m \in[0.5,1)$.

2) $\theta(t)$ is uniformly distributed over $[0,2 \pi)$.

3) $r(t)$ and $\theta(t)$ are mutually independent.

The probability density function (pdf) of $r(t)$ for an arbitrary time $t$ is given by [2]

$$
p(r(t)=r)= \begin{cases}\frac{2}{\Gamma(m)}\left(\frac{m}{\Omega}\right)^{m} r^{2 m-1} e^{-m r^{2} / \Omega}, & \geq 0 \\ 0, & r<0\end{cases}
$$

where $\Gamma(\cdot)$ is the gamma function and $m$ is in the range $0.5 \leq$ $m<1$. Our task is to develop a simulation model that can be used to realize $z(t)$. In the development of this model, the following lemma is required.

Lemma 1: Let $r_{z}$ be an $m$ distributed random variable with the second moment $E\left\{r_{z}^{2}\right\}=\Omega$ and the fading parameter $m \in$ $[0.5,1)$. Let $r_{w}$ be a Rayleigh distributed random variable with $E\left\{r_{w}^{2}\right\}=\Omega / m$. If $r_{z}$ and $r_{w}$ satisfy the functional relationship

$$
r_{z}=\sqrt{\xi} r_{w}
$$

where $\xi$ is a nonnegative random variable independent of $r_{w}$, then $\xi$ has a standard beta distribution with parameters $m$ and $1-m$. The pdf of $\xi$ is given by [12]

$p(\xi)= \begin{cases}\frac{1}{B(m, 1-m)} \xi^{m-1}(1-\xi)^{(1-m)-1}, & 0<\xi<1 \\ 0, & \text { otherwise }\end{cases}$

where $B(a, b)=\Gamma(a) \Gamma(b) / \Gamma(a+b)$ is a beta function.

The proof is given in Appendix A. Let $\theta$ be a random variable uniformly distributed over $[0,2 \pi)$ and independent of $r_{z}, r_{w}$, and $\xi$. It follows that $w=r_{w} e^{j \theta}$ is a zero-mean complex Gaussian random variable with uncorrelated real and imaginary parts. Multiplying both sides of (3) by $e^{j \theta}$ gives $r_{z} e^{j \theta}=\xi^{1 / 2} w$. This expression implies that $z(t)$ can be represented by

$$
z(t)=\mu(t) w(t)
$$

where i) $\mu(t)$ is a WSS nonnegative random process and $\mu^{2}(t)$ follows a standard beta distribution with parameters $m$ and $1-$ $m$, and ii) $w(t)$, being independent of $\mu(t)$, is a zero-mean WSS complex Gaussian process with uncorrelated real and imaginary parts and with an autocorrelation function

$$
R_{w}(\Delta t)=E\left\{w(t) w^{*}(t+\Delta t)\right\}
$$

satisfying $R_{w}(0)=\Omega / m$. Notice that $R_{z}(\Delta t)$ and $R_{w}(\Delta t)$ are related by

$$
R_{z}(\Delta t)=R_{\mu}(\Delta t) \cdot R_{w}(\Delta t)
$$

where

$$
R_{\mu}(\Delta t)=E\{\mu(t) \mu(t+\Delta t)\}
$$

and that $R_{\mu}(0)=m$. The representation of $z(t)$ by (5) is the desired simulation model. One can generate $z(t)$ by generating a complex Gaussian process $w(t)$ and an appropriate square-root-beta random process $\mu(t)$, followed by substituting $w(t)$ and $\mu(t)$ into (5). The complex Gaussian process $w(t)$ can be generated by an established technique, e.g., [8]. The non-Gaussian process $\mu(t)$ can be generated by a nonlinear transformation of a Gaussian process [13]. Appendix B shows that

$$
\mu(t)=F^{-1}(\Phi(y(t)))
$$

where i) $y(t)$ is a zero-mean unit-variance Gaussian process with an autocorrelation function

$$
\rho(\Delta t)=E\{y(t) y(t+\Delta t)\}
$$

and ii) $F$ and $\Phi$ are defined by (23) and (24), respectively, and $F^{-1}$ is the inverse of $F$. The relationship between $\rho(\Delta t)$ and $R_{\mu}(\Delta t)$ is given by (26a) and (26b).

\section{B. Extension for Simulation of Correlated Diversity Channels}

Antenna diversity is a well-known technique in HF communications for combating adverse effects due to fading. So far our discussion has been concentrated on simulating an $m$ fading channel by generating a complex random process $z(t)$. This approach is useful for simulating a communication system with antenna diversity only if the diversity branches are independently faded. When the antenna spacing is inadequate, nonindependent fading among diversity branches occurs so that the $m$ fading channels used for modeling the diversity channels are correlated. It gives rise to the need for simulating correlated diversity channels. In what follows, we demonstrate how to simulate correlated $m$ fading channels for $m<1$ by a simple extension of the proposed simulation model.

Let $M$ be the order of diversity. We assume that the $M$ diversity channels have the same fading parameter $m$, second moment $\Omega$, and autocorrelation function $R_{z}(\Delta t)$. This assumption is appropriate for a communication system using antenna diversity. In the implementation of simulation, one is given a 
knowledge of the correlation matrix $\rho=\left[\rho_{k, \ell}\right]_{k, \ell=1}^{M}$ that characterizes the correlation among the $M$ diversity channels, where $\left|\rho_{k, \ell}\right| \leq 1, \rho_{k, k}=1$ and $\rho$ is real symmetric. To simulate $M$ correlated channels, it is required to generate $M$ correlated complex processes $z_{k}(t), k=1, \cdots, M$, each of which has the properties of $z(t)$ specified in Section II-A. Note that

$$
\rho_{k, \ell}=\frac{E\left\{z_{k}(t) z_{\ell}^{*}(t)\right\}}{\sqrt{E\left\{\left|z_{k}(t)\right|^{2}\right\} \cdot E\left\{\left|z_{\ell}(t)\right|^{2}\right\}}}
$$

and that $\rho$ is nonnegative definite ([14], p. 295). Applying the proposed model (5) to the situation under consideration, we have

$$
z_{k}(t)=\mu_{k}(t) w_{k}(t), \quad k=1, \cdots, M
$$

where i) $\mu_{k}(t)$ is a WSS nonnegative random process and $\mu_{k}^{2}(t)$ has a standard beta distribution with parameters $m$ and $1-m$, and ii) $w_{k}(t)$, being independent of $\mu_{k}(t)$, is a zero-mean WSS complex Gaussian process with uncorrelated real and imaginary parts and with a variance given by $\Omega / m$. We assume that i) any pair of $\mu_{k}(t)$ and $w_{\ell}(t), k, \ell=1, \cdots, M$ are independent, and ii) $\mu_{k}(t)$ and $\mu_{\ell}(t), k \neq \ell$ are mutually independent. Assumption $i)$ is made because it makes implementation easier and does not affect resultant statistical properties of $z_{k}(t)$ 's. Assumption ii) leads to conditions that are usually satisfied in practice [see comments after (16)]. Substituting (12) into (11) yields

$$
\rho_{k, \ell}= \begin{cases}K_{m} \rho_{k, \ell}^{(w)}, & k \neq \ell \\ \rho_{k, \ell}^{(w)}, & k=\ell\end{cases}
$$

where

$$
\rho_{k, \ell}^{(w)}=\frac{E\left\{w_{k}(t) w_{\ell}^{*}(t)\right\}}{\sqrt{E\left\{\left|w_{k}(t)\right|^{2}\right\} \cdot E\left\{\left|w_{\ell}(t)\right|^{2}\right\}}}
$$

and

$$
K_{m}=\frac{E\left\{\mu_{k}(t)\right\} \times E\left\{\mu_{\ell}(t)\right\}}{\sqrt{E\left\{\mu_{k}^{2}(t)\right\} \cdot E\left\{\mu_{\ell}^{2}(t)\right\}}}=\frac{4}{\pi m}\left[\frac{\Gamma\left(m+\frac{1}{2}\right)}{\Gamma(m)}\right]^{2} .
$$

With a knowledge of $\rho_{k, \ell}$, one can determine $\rho_{k, \ell}^{(w)}$ by (13). Since autocorrelation functions of $z_{k}(t)$ are assumed the same for all $k$ 's and are given by $R_{z}(\Delta t)$, for convenience in the implementation, we assume that autocorrelation functions of $\mu_{k}(t)$ 's and $w_{k}(t)$ 's are given by $R_{\mu}(\Delta t)$ and $R_{w}(\Delta t)$, respectively, both of which are independent of $k$. Note that $R_{z}(\Delta t), R_{\mu}(\Delta t)$ and $R_{w}(\Delta t)$ are related by (7). After $R_{\mu}(\Delta t)$ is determined, one can generate each of $\mu_{k}(t), k=1, \cdots, M$, by a nonlinear transformation of a Gaussian process as shown in Appendix B. Generation of $\mathbf{w}(t)=\left[w_{1}(t), \cdots, w_{M}(t)\right]^{T}$ can be accomplished by the following method. Let $u_{k}(t), k=1, \cdots, M$, be mutually independent zero-mean complex Gaussian processes, each of which has an autocorrelation function $R_{w}(\Delta t)$ and uncorrelated real and imaginary parts. Each of $u_{k}(t)$ 's can be gener- ated by a known method. Let $\mathbf{u}(t)=\left[u_{1}(t), \cdots, u_{M}(t)\right]^{T}$ and $\rho^{(w)}=\left[\rho_{k, \ell}^{(w)}\right]_{k, \ell=1}^{M}$. Then

$$
\mathbf{w}(t)=\mathbf{C u}(t)
$$

where $\mathbf{C}$ is a factor of $\rho^{(w)}$ such that $\mathbf{C} \mathbf{C}^{T}=\rho^{(w)}$.

To generate $\mathbf{w}(t)$, the matrix $\mathbf{C}$ must exist, which requires that $\rho^{(w)}$ is nonnegative definite. That is, for any $a_{1}, a_{2}, \cdots, a_{M}$ with at least one of which is nonzero, the condition $\sum_{k=1}^{M} \sum_{\ell=1}^{M} a_{k} a_{\ell} \rho_{k, \ell}^{(w)} \geq 0$ needs to be satisfied. Substituting (13) into this requirement yields

$$
\sum_{k=1}^{M} \sum_{\ell=1}^{M} a_{k} a_{\ell} \rho_{k, \ell}-\left(1-K_{m}\right) \sum_{k=1}^{M} a_{k}^{2} \geq 0 .
$$

It is known that (16) can be satisfied if $1-K_{m}$ is less than the smallest eigenvalue of $\rho$ [14, Ch. 11.10]. As a practical example, consider a dual-diversity system $(M=2)$. The smallest eigenvalue is $1-\rho_{1,2}$, so that (16) is satisfied if $\rho_{1,2} \leq K_{m}$. It can be shown that the minimum value of $K_{m}$ over the range $m \in[0.5,1)$ is 0.8106 . As the correlation between diversity channels seldom exceeds 0.8106 for practical cases of interest, (16) is usually satisfied. For diversity channels with $1-K_{m}$ greater than the smallest eigenvalue of $\rho, z_{k}(t)$ 's can be generated by allowing $\mu_{k}(t)$ 's to be correlated.

\section{IMPLEMENTATION FOR SIMULATION}

It is desired to realize a complex random process $z(t)$ in order to simulate an $m$ fading channel, $m<1$, with a given Doppler power spectrum $S_{z}(f)$. The power spectrum $S_{z}(f)$ is related to $R_{z}(\Delta t)$ by

$$
S_{z}(f)=\int_{-\infty}^{\infty} e^{-j 2 \pi f \Delta t} R_{z}(\Delta t) d \Delta t .
$$

Since Doppler power spectra of HF channels that are of practical interest are band-limited, we consider only the case of generating a band-limited process $z(t)$. Let $f_{\max }$ be the frequency such that $S_{z}(f)$ is zero or negligible for $|f|>f_{\max }$. To realize $z(t)$, one can generate a sequence of equidistant discrete-time samples $z\left(n T_{s}\right), T_{s}=\left(\alpha f_{\max }\right)^{-1}$ and $n=0, \pm 1, \pm 2, \cdots$, followed by interpolation to obtain the value of $z(t)$ for an arbitrary time $t$, where $\alpha \geq 2$ is the oversampling factor. A value of $\alpha \geq 8$ is usually sufficient to generate $z(t)$ without appreciable loss of accuracy [4]. After the value of $\alpha$ is determined, the rest of the procedure except interpolation directly follows from an application of the proposed simulation model.

The procedure is given as follows.

1) Compute $R_{z}\left(\Delta n \cdot T_{s}\right), \Delta n=-N, \cdots, N$, by (17), where $N$ is a a sufficiently large integer. This sequence of $R_{z}\left(\Delta n \cdot T_{s}\right)$ is used in generating $z\left(n T_{s}\right)$ 's so that a greater value of $N$ improves the accuracy of the autocorrelation property of the generated $z\left(n T_{s}\right)$ 's, although more computation is required.

2) Select an appropriate $\rho(\Delta t)$. Since (7) implies that $S_{z}(f)$ is given by the convolution between the power spectrum of $\mu(t)$ and that of $w(t)$, and since $z(t)$ is band-limited to $f_{\max }$, it follows that $\mu(t)$ and $w(t)$ are band-limited to at most $f_{\max }$. As $\mu(t)$ is generated from $y(t)$ by (9), 
TABLE I

$R_{\mu}(\Delta t) / R_{\mu}(0)$ VALUes Against $\rho(\Delta t)$ FOR $0.5 \leq m<1$. Note THAT $R_{\mu}(0)=m$

\begin{tabular}{ccccccccccc}
\hline$\rho(\Delta t)$ & & & \multicolumn{9}{c}{$R_{\mu}(\Delta t) / R_{\mu}(0)$} \\
& $m=0.5$ & 0.55 & 0.6 & 0.65 & 0.7 & 0.75 & 0.8 & 0.85 & 0.9 & 0.95 \\
\hline-1.0 & 0.6366 & 0.7028 & 0.7612 & 0.8122 & 0.8562 & 0.8936 & 0.9248 & 0.9503 & 0.9708 & 0.9871 \\
-0.9 & 0.6550 & 0.7162 & 0.7704 & 0.8180 & 0.8594 & 0.8951 & 0.9253 & 0.9504 & 0.9708 & 0.9871 \\
-0.8 & 0.6728 & 0.7297 & 0.7801 & 0.8245 & 0.8634 & 0.8972 & 0.9262 & 0.9507 & 0.9709 & 0.9871 \\
-0.7 & 0.6906 & 0.7433 & 0.7901 & 0.8314 & 0.8679 & 0.8998 & 0.9275 & 0.9511 & 0.9710 & 0.9871 \\
-0.6 & 0.7096 & 0.7569 & 0.8003 & 0.8388 & 0.8728 & 0.9028 & 0.9291 & 0.9518 & 0.9711 & 0.9871 \\
-0.5 & 0.7252 & 0.7706 & 0.8108 & 0.8465 & 0.8782 & 0.9063 & 0.9311 & 0.9527 & 0.9714 & 0.9872 \\
-0.4 & 0.7424 & 0.7843 & 0.8215 & 0.8545 & 0.8840 & 0.9101 & 0.9334 & 0.9539 & 0.9719 & 0.9872 \\
-0.3 & 0.7594 & 0.7980 & 0.8323 & 0.8628 & 0.8900 & 0.9143 & 0.9360 & 0.9553 & 0.9725 & 0.9874 \\
-0.2 & 0.7764 & 0.8119 & 0.8434 & 0.8714 & 0.8964 & 0.9188 & 0.9390 & 0.9570 & 0.9732 & 0.9875 \\
-0.1 & 0.7934 & 0.8259 & 0.8546 & 0.8802 & 0.9031 & 0.9237 & 0.9422 & 0.9590 & 0.9741 & 0.9878 \\
0.0 & 0.8106 & 0.8400 & 0.8660 & 0.8893 & 0.9101 & 0.9288 & 0.9458 & 0.9612 & 0.9753 & 0.9881 \\
0.1 & 0.8278 & 0.8543 & 0.8777 & 0.8986 & 0.9173 & 0.9342 & 0.9496 & 0.9636 & 0.9766 & 0.9886 \\
0.2 & 0.8452 & 0.8688 & 0.8896 & 0.9082 & 0.9249 & 0.9400 & 0.9537 & 0.9663 & 0.9781 & 0.9891 \\
0.3 & 0.8628 & 0.8835 & 0.9018 & 0.9181 & 0.9328 & 0.9460 & 0.9581 & 0.9693 & 0.9798 & 0.9898 \\
0.4 & 0.8808 & 0.8986 & 0.9143 & 0.9284 & 0.9410 & 0.9524 & 0.9629 & 0.9726 & 0.9818 & 0.9907 \\
0.5 & 0.8990 & 0.9140 & 0.9272 & 0.9390 & 0.9496 & 0.9592 & 0.9680 & 0.9762 & 0.9840 & 0.9916 \\
0.6 & 0.9178 & 0.9299 & 0.9405 & 0.9500 & 0.9586 & 0.9663 & 0.9734 & 0.9801 & 0.9865 & 0.9928 \\
0.7 & 0.9370 & 0.9463 & 0.9544 & 0.9615 & 0.9680 & 0.9739 & 0.9793 & 0.9843 & 0.9892 & 0.9942 \\
0.8 & 0.9570 & 0.9633 & 0.9688 & 0.9736 & 0.9780 & 0.9819 & 0.9856 & 0.9890 & 0.9924 & 0.9958 \\
0.9 & 0.9780 & 0.9812 & 0.9839 & 0.9864 & 0.9886 & 0.9906 & 0.9924 & 0.9942 & 0.9959 & 0.9977 \\
1.0 & 1.0000 & 1.0000 & 1.0000 & 1.0000 & 1.0000 & 1.0000 & 1.0000 & 1.0000 & 1.0000 & 1.0000 \\
\hline
\end{tabular}

$y(t)$ is most often band-limited to at most $f_{\max }$. It is recommended that a convenient choice of $\rho(\Delta t)$ is selected, where

$$
S_{y}(f)=\int_{-\infty}^{\infty} e^{-j 2 \pi f \Delta t} \rho(\Delta t) d \Delta t
$$

vanishes or is negligible for $|f|>f_{\max }$.

3) Compute $R_{\mu}\left(\Delta n \cdot T_{s}\right)$ from $\rho\left(\Delta n \cdot T_{s}\right)$ by (26a) and (26b) or with the aid of Table I, which lists the $R_{\mu}(\Delta t) / R_{\mu}(0)$ values against $\rho(\Delta t)$. Then compute $R_{w}\left(\Delta n \cdot T_{s}\right)$ by (7). To ensure that $w(t)$ can be realized, $R_{w}(\Delta t)$ is required to satisfy that $\left|R_{w}(\Delta t)\right| \leq R_{w}(0)$ and $S_{w}(f) \geq 0$ for all $f$, where

$$
S_{w}(f)=\int_{-\infty}^{\infty} e^{-j 2 \pi f \Delta t} R_{w}(\Delta t) d \Delta t
$$

is the power spectrum of $w(t)$. If the selected $\rho(\Delta t)$ cannot yield $R_{w}\left(\Delta n \cdot T_{s}\right)$ 's that satisfy $\left|R_{w}\left(\Delta n \cdot T_{s}\right)\right| \leq R_{w}(0)$, another choice of $\rho(\Delta t)$ should be made, and Step 2) should be repeated. However, selection of $\rho(\Delta t)$ that satisfies $S_{w}(f) \geq 0$ for all $f$ is rather difficult. Appendix C shows that $S_{w}(f)$ can be computed by (20), shown at the bottom of the page. For a given $\rho(\Delta t)$, it is possible that the computed $S_{w}(f)$ is negative for some $f$, although the contribution of this negative part to the total $S_{w}(f)$ is usually not significant. In addition, $S_{w}(f)$ computed by (20) is not limited for $|f| \leq f_{\text {max }}$. Instead of carrying out the difficult task to find a particular $\rho(\Delta t)$ that makes $S_{w}(f) \geq 0$ for all $f$, it is recommended that $S_{w}(f)$ may be modified by the following steps.

a) Set $S_{w}(f)=0$ wherever $S_{w}(f)$ computed by (20) is negative.

b) Set $S_{w}(f)=0$ for $|f|>f_{\max }$.

c) Scale the resultant $S_{w}(f)$ such that $\int_{-\infty}^{\infty} S_{w}(f) d f=R_{w}(0)=\Omega / m$.

Although autocorrelation properties of the generated $z(t)$ deviate from the desired ones as a result of this modification, numerical results of Section IV demonstrate that this deviation is small. The modified $S_{w}(f)$ is used to compute new values of $R_{w}\left(\Delta n \cdot T_{s}\right)$.

4) Based on the selected $\rho(\Delta t)$ or $S_{y}(f)$, generate Gaussiandistributed samples $y\left(n T_{s}\right)$ by a known method. A computationally efficient method is preferred. Then generate $\mu\left(n T_{s}\right)$ from the generated $y\left(n T_{s}\right)$ by (9). In (9), although a closed-form expression of $F^{-1}(\Phi(\cdot))$ is available only for $m=0.5$ (see Appendix B), for other values of $m$, numerical routines are easily implemented for computation of this function.

5) Generate complex-Gaussian distributed samples $w\left(n T_{s}\right)$ based on the modified $S_{w}(f)$ or the new values of $R_{w}\left(\Delta n \cdot T_{s}\right)$. Again, an efficient technique is preferred.

6) Compute $z\left(n T_{s}\right)$ by (5).

To simulate correlated diversity channels, one is required to generate $\mathbf{z}(t)=\left[z_{1}(t), \cdots, z_{M}(t)\right]^{T}$ where each of $z_{1}(t), \cdots, z_{M}(t)$ has a Doppler power spectrum $S_{z}(f)$ given by (17). A sequence of equidistant discrete-time samples $\mathbf{z}\left(n T_{s}\right), T_{s}=\left(\alpha f_{\max }\right)^{-1}$ and $n=0, \pm 1, \pm 2, \cdots$ is first

$$
S_{w}(f)= \begin{cases}T_{s}\left\{R_{w}(0)+2 \sum_{\Delta n=1}^{N} \operatorname{Re}\left[R_{w}\left(\Delta n \cdot T_{s}\right) e^{-j 2 \pi f \Delta n \cdot T_{s}}\right]\right\}, & |f| \leq 1 /\left(2 T_{s}\right) \\ 0, & \text { otherwise }\end{cases}
$$


generated, followed by interpolation to obtain the value of $\mathbf{z}(t)$ for an arbitrary time $t$. The procedure for generating $\mathbf{z}\left(n T_{s}\right)$ follows from the one for generating $z\left(n T_{s}\right)$ described above, except that an additional step is required to compute $\mathrm{w}\left(n T_{s}\right)$ from the generated $\mathbf{u}\left(n T_{s}\right)$ by (15). In applying (15), different factorization methods can be used. However, Cholesky factorization is preferred. It gives a lower triangular matrix $\mathbf{C}$ so that some of the steps in multiplication and addition can be eliminated in simulation. This reduces the computation cost.

\section{NUMERICAL EXAMPLES}

\section{A. Example 1}

A realization of $z(t)$ was generated based on the procedure given in Section III. For illustration purposes, we assumed that $m=0.5$ and $\rho(\Delta t)=1$. A double-Gaussian spectrum was used

$$
\begin{aligned}
S_{z}(f)= & \frac{1}{2 \sqrt{2 \pi} f_{\Delta}}\left\{\exp \left[-\frac{1}{2}\left(\frac{f-\gamma f_{\max }}{f_{\Delta}}\right)^{2}\right]\right. \\
& \left.+\exp \left[-\frac{1}{2}\left(\frac{f+\gamma f_{\max }}{f_{\Delta}}\right)^{2}\right]\right\} \\
\leftrightarrow R_{z}(\Delta t)= & \cos \left(2 \pi \gamma f_{\max } \Delta t\right) \times \exp \left[-2\left(\pi f_{\Delta} t\right)^{2}\right]
\end{aligned}
$$

where $f_{\Delta}=(1-\gamma) f_{\max } / 3$ and we arbitrarily selected $\gamma=0.5$. This value of $f_{\Delta}$ was chosen in order to ensure that $S_{z}(f)$ is negligible for $|f|>f_{\max }$, so that the Doppler power spectrum under consideration can be considered band-limited. The double-Gaussian spectrum was selected because it is appropriate for modeling Doppler power spectra of HF channels [1]. Although a symmetric spectrum is used here for simplicity, realistic spectra for HF channels are usually asymmetric [1]. An oversampling ratio of $\alpha=10$ was employed.

We first computed $R_{z}\left(\Delta n \cdot T_{s}\right), \Delta n=-N, \cdots, N$, where $N=1000$ was arbitrarily chosen. For the sake of convenience, we selected

$$
\begin{aligned}
S_{y}(f) & =\frac{1}{\sqrt{2 \pi} f_{y}} \exp \left[-\frac{1}{2}\left(f / f_{y}\right)^{2}\right] \\
\leftrightarrow \rho(\Delta t) & =\exp \left[-2\left(\pi f_{y} \Delta t\right)^{2}\right]
\end{aligned}
$$

where $f_{y}=f_{\max } / 3$ was used so that $S_{w}(f)$ is negligible for $|f|>f_{\max }$. Based on the selected $\rho(\Delta t)$ and values of $R_{z}(\Delta n$. $T_{s}$ ), we computed $R_{\mu}\left(\Delta n \cdot T_{s}\right)$ and $R_{w}\left(\Delta n \cdot T_{s}\right)$. Then $S_{w}(f)$ was computed from $R_{w}\left(\Delta n \cdot T_{s}\right)$ values by (20). For $|f|>f_{\max }$ and at frequencies where computed $S_{w}(f)$ values were negative, we set $S_{w}(f)=0$. The resultant $S_{w}(f)$ was scaled so that $\int_{-\infty}^{\infty} S_{w}(f) d f=\Omega / m=2$. It was found that $3.26 \%$ of the original $S_{w}(f)$ were truncated. A total of $10^{4}$ discrete-time samples $z\left(n T_{s}\right)$ were generated by the following procedure. The Gaussian-distributed time series $y\left(n T_{s}\right)$ was generated by an efficient technique based on Rice's sum of sinusoids [8]. The time series $\mu\left(n T_{s}\right)$ was generated from the time series $y\left(n T_{s}\right)$ by the nonlinear transformation given by (9). The complex Gaussian time series $w\left(n T_{s}\right)$ was generated by separately generating the in-phase and quadrature-phase components based on the sum of sinusoids method. Parameters of the sum of sinusoids method used in generating $y\left(n T_{s}\right)$ and $w\left(n T_{s}\right)$ were computed based on the procedure of [8]. ${ }^{1}$ The value of $z\left(n T_{s}\right)$ was computed from $\mu\left(n T_{s}\right)$ and $w\left(n T_{s}\right)$ by (5). After $z\left(n T_{s}\right)$ 's were generated, $z\left(\left(n+(1 / 2) T_{s}\right), n=1, \cdots, 10^{4}\right.$, were computed by piecewise-constant interpolation. A total of $2 \times 10^{4}$ samples, i.e., $z\left(n T_{\text {sim }}\right), n=2, \cdots,\left(2 \times 10^{4}+1\right)$, where $T_{\text {sim }}=T_{s} / 2$, were finally obtained.

Fig. 1(a) and (b) plot the cumulative distribution functions (cdf's) of the amplitude and phase, respectively, computed from the $2 \times 10^{4}$ generated $z\left(n T_{\text {sim }}\right)$ samples. The cdf of the $m$ distribution with $m=0.5$ and $\Omega=1$, and that of the uniform distribution for the range $[0,2 \pi)$, are also plotted for comparison purposes. It is apparent that the distributions of the generated samples fit closely to the desired ones. Fig. 1(c) plots the autocorrelation function computed from the generated samples and the one for the double-Gaussian spectrum. It is shown that the generated autocorrelation function is close to the desired one. The result indicates that despite the need to truncate the original $S_{w}(f)$, the loss of accuracy in the autocorrelation property of the generated samples is small.

\section{B. Example 2}

In this example, we consider simulating a dual-branch $(M=$ 2 ) diversity channel with correlated fading by generating $\mathbf{z}(t)=$ $\left[z_{1}(t), z_{2}(t)\right]^{T}$ using (12). It is assumed that the correlation between the two branches is $\rho_{1,2}=\rho_{2,1}=0.6$ and that the fading parameter is $m=0.9$. These two values are arbitrarily selected for illustration purpose. Furthermore, we assume that $\Omega=1, \alpha=10$ and $S_{z}(f)$ is given by (21) with $\gamma=0.5$.

We generated $10^{4}$ samples of $\mathbf{z}\left(n T_{s}\right)$ by generating $\mu\left(n T_{s}\right)$ and $\mathbf{w}\left(n T_{s}\right)$ where $\mu(t)=\left[\mu_{1}(t), \mu_{2}(t)\right]^{T}$ and $\mathbf{w}(t)=\left[w_{1}(t), w_{2}(t)\right]^{T}$. Generation of each of the independent time series $\mu_{1}\left(n T_{s}\right)$ and $\mu_{2}\left(n T_{s}\right)$ followed the same procedure as in generating $\mu\left(n T_{s}\right)$ in Example 1. The same $\rho(\Delta t)$, given by (22) was used in generating $\mu_{1}\left(n T_{s}\right)$ and $\mu_{2}\left(n T_{s}\right)$. As a result of this particular choice of $\rho(\Delta t)$, $0.20 \%$ of the original $S_{w}(f)$ needed to be truncated. The $\mathbf{w}\left(n T_{s}\right)$ was generated by (15). Generation of each of the time series $u_{1}\left(n T_{s}\right)$ and $u_{2}\left(n T_{s}\right)$ also followed the procedure of generating $w\left(n T_{s}\right)$ in Example 1. The time series $\mathbf{z}\left(n T_{s}\right)$ was obtained by substituting the generated $\mu\left(n T_{s}\right)$ and $\mathbf{w}\left(n T_{s}\right)$ into (12). After the time series $\mathbf{z}\left(n T_{s}\right)$ was generated, $\mathbf{z}\left((n+(1 / 2)) T_{s}\right), n=1, \cdots, 10^{4}$, were computed by piecewise-constant interpolation. A total of $2 \times 10^{4}$ samples of $\mathbf{z}\left(n T_{\text {sim }}\right), T_{\text {sim }}=T_{s} / 2$, were obtained.

Fig. 2(a)-(c) plot the cdf's of amplitude, phase, and autocorrelation functions, respectively, computed from each set of $2 \times 10^{4}$ generated samples $z_{k}\left(n T_{\text {sim }}\right), k=1,2$. The desired cdf's and autocorrelation functions are also plotted. It is apparent that computed autocorrelation functions and cdf's are

${ }^{1}$ One of the reviewers pointed out that statistical properties of $\left\{y\left(n T_{s}\right)\right\}$ and $\left\{w\left(n T_{s}\right)\right\}$ generated by the sum of sinusoids method can be verified analytically by the closed-form expressions of [8], so that the autocorrelation and phase distribution of the generated $\left\{z\left(n T_{s}\right)\right\}$ can be verified before $z\left(n T_{s}\right)$ 's are actually generated. This advantage enables more rapid verification of the simulation accuracy for the selected set of parameters. 


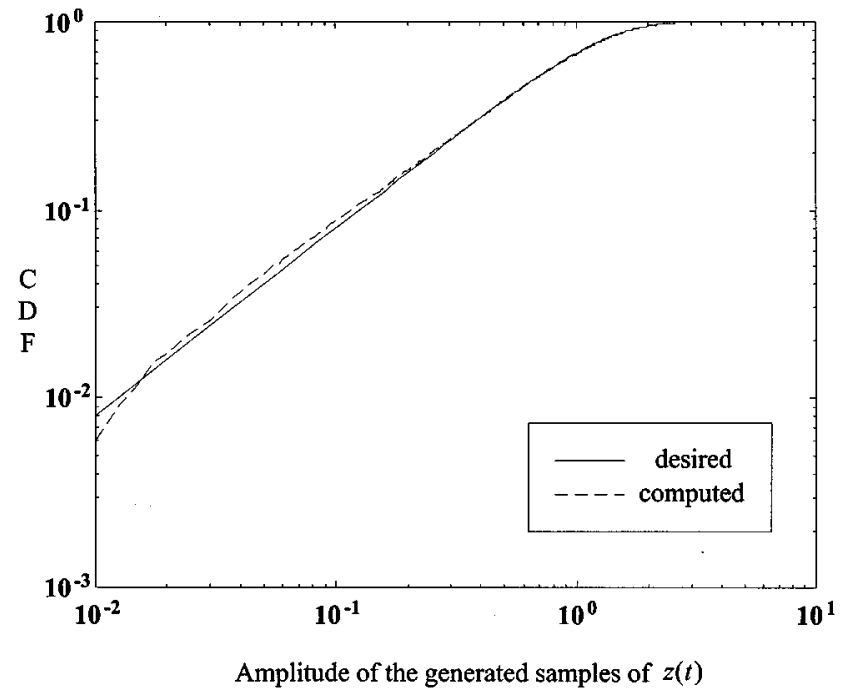

(a)

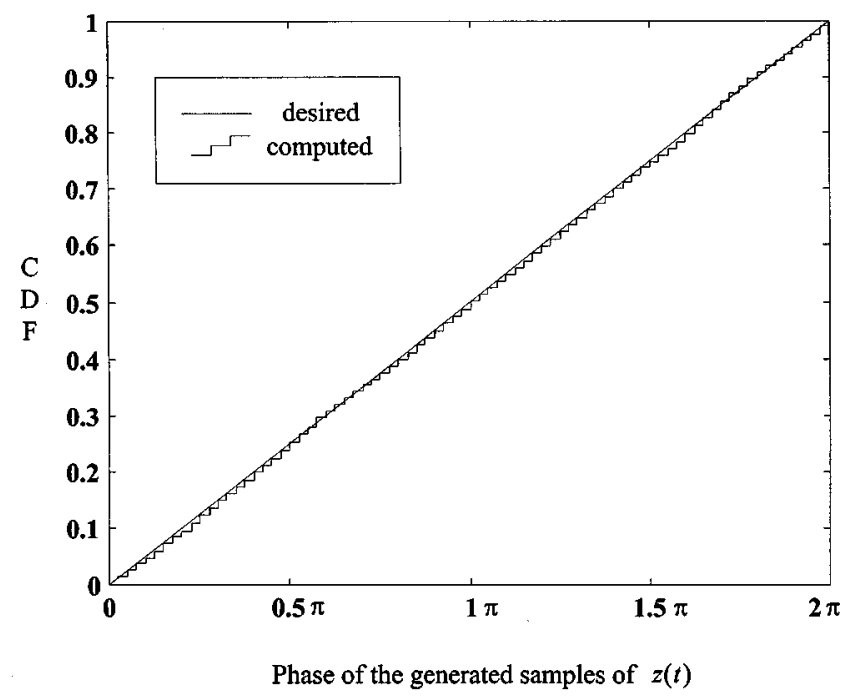

(b)

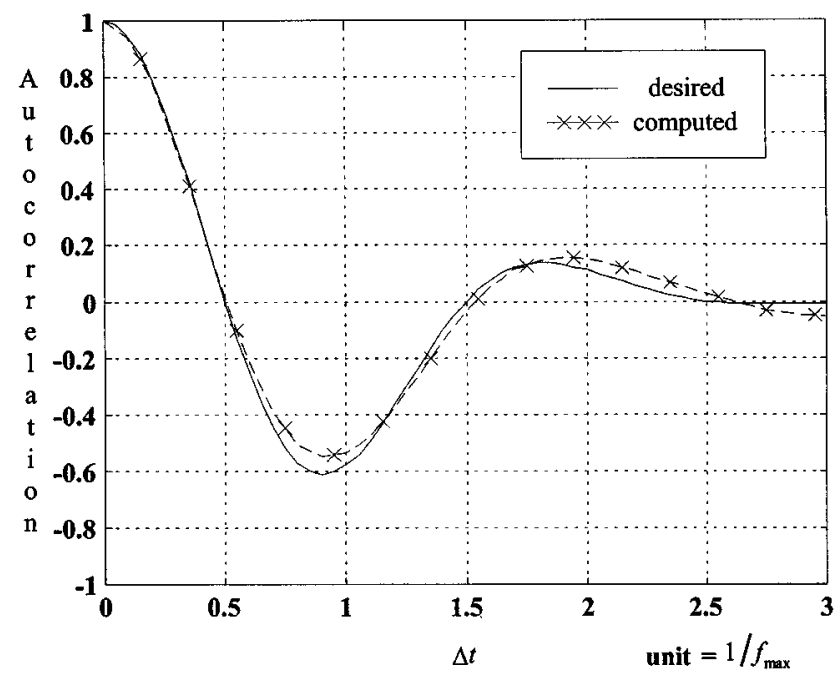

(c)

Fig. 1. Statistics computed from the generated samples of $z(t)$ in Example 1: (a) cdf of amplitude, (b) cdf of phase, and (c) autocorrelation function. The desired cdf's and autocorrelation function are also plotted.

close to the desired ones. We also computed the correlation matrix $\hat{\rho}$ from the generated time series $z_{1}\left(n T_{\text {sim }}\right)$ and $z_{2}\left(n T_{\text {sim }}\right)$ and found that

$$
\hat{\rho}=\left[\begin{array}{cc}
1.0000 & 0.6016+0.0289 j \\
0.6016-0.0289 j & 1.0000
\end{array}\right] .
$$

In comparison with the desired correlation matrix $\rho\left(\rho_{1,2}=\right.$ $\rho_{2,1}=0.6$ and $\left.\rho_{1,1}=\rho_{2,2}=1\right)$, it is apparent that the computed correlation matrix $\hat{\rho}$ is close to $\rho$.

\section{CONCLUSION}

We have proposed a simulation model for $m$ fading channels, $m<1$. The model reveals that an $m$ fading channel, $m<1$, having a given Doppler power spectrum can be simulated by generating a complex Gaussian process and a square-root-beta random process. Application of the proposed model to simulate correlated $m$ fading channels has also been demonstrated. In numerical examples, it has been shown that statistical properties of the samples generated from the use of the proposed model are close to the required ones.

\section{APPENDIX A}

\section{PROOF OF LEMMA 1}

Let $c_{z}=r_{z}^{2}$ and $c_{w}=r_{w}^{2}$. The proof can be accomplished by taking Mellin transform on both sides of $c_{z}=\xi c_{w}$ and noting that the Mellin transform of $\xi c_{w}$ is the product of the Mellin transform of $\xi$ and that of $c_{w}$. An alternative proof can also be obtained by using the fact that $c_{z}$ and $c_{w}$ are gamma random variables and that a beta random variable can be expressed as a ratio of two gamma random variables [12, Ch. 25.2]. 


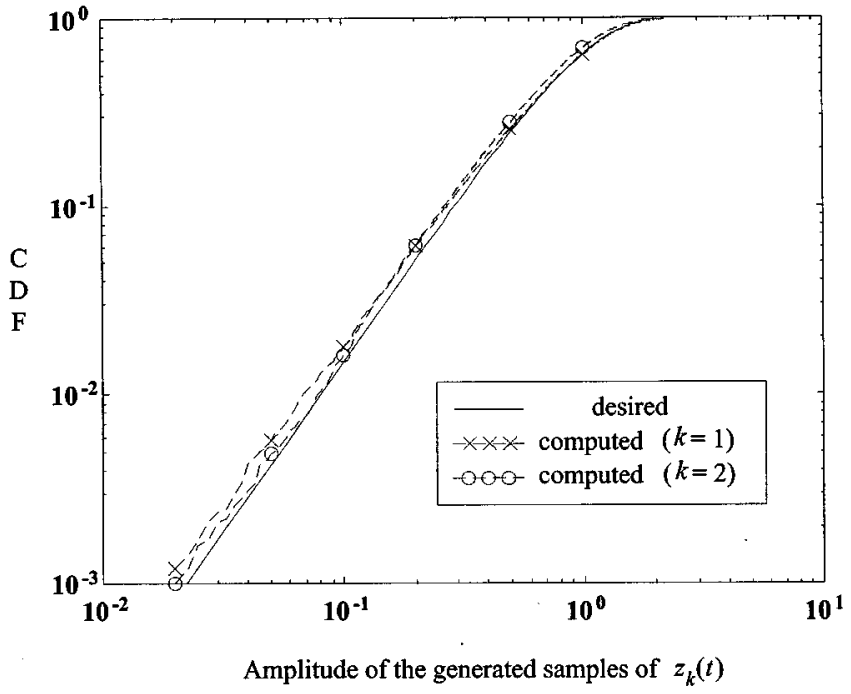

(a)

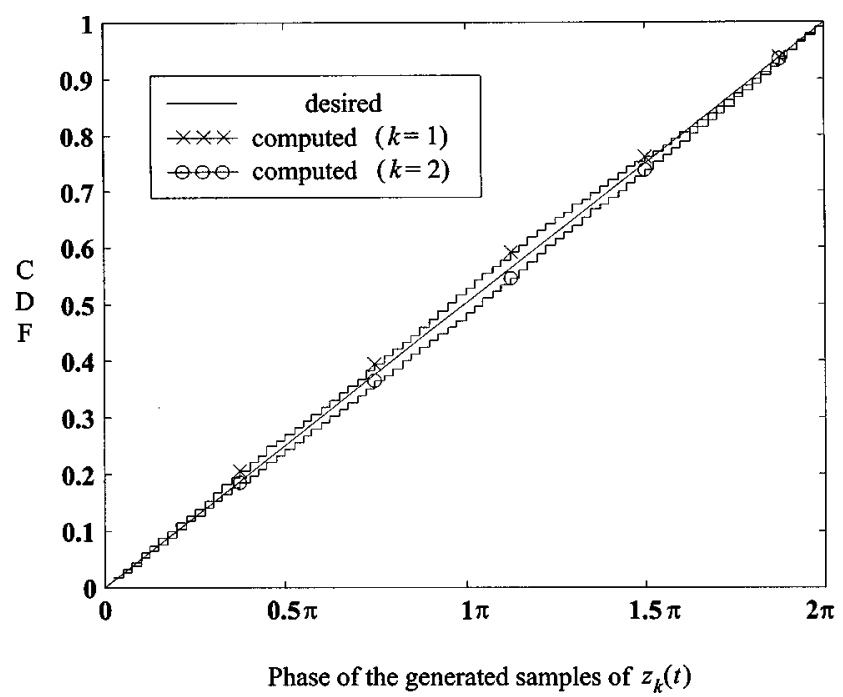

(b)

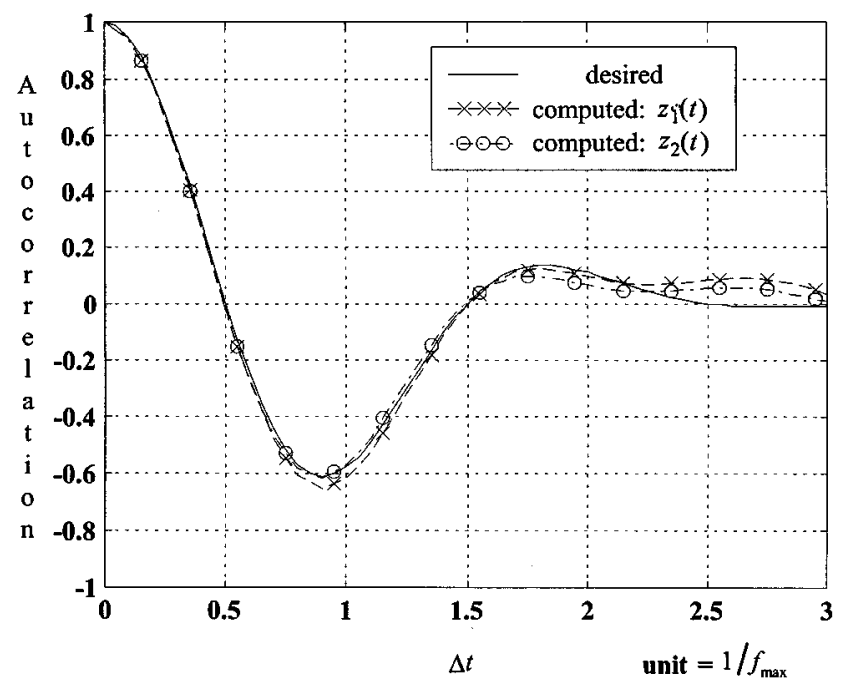

(c)

Fig. 2. Statistics computed from the generated samples of $z_{1}(t)$ and $z_{2}(t)$ in Example 2: (a) cdf's of amplitude, (b) cdf's of phase, and (c) cdf's of autocorrelation functions. The desired cdf's and autocorrelation function are also plotted.

\section{APPENDIX B \\ REALIZATION OF $\mu(t)$}

Let $F(x)$ be the cdf of a square-root-beta random variable with parameters $m$ and $1-m$, and let $\Phi(x)$ be the cdf of a standard normal random variable. It follows that

$$
F(x)=I_{x^{2}}(m, 1-m), \quad 0 \leq x \leq 1
$$

and

$$
\Phi(x)=(2 \pi)^{-1 / 2} \int_{-\infty}^{x} e^{-t^{2} / 2} d t
$$

respectively, where $I_{u}(a, b)=(B(a, b))^{-1} \int_{0}^{u} t^{a-1}(1-t)^{b-1} d t$ is the incomplete beta function. Note that in the special case of $m=0.5, F(x)=(2 / \pi) \sin ^{-1} x$ for $0 \leq x \leq 1$. It is easy to show that $F(x)$ is a strictly increasing function for $0 \leq x \leq 1$ and is bounded by $F(x) \in[0,1]$. If we specify that $F^{-1}(v)$, the inverse of $F$, gives a value within $[0,1]$ for $0 \leq v \leq 1$, then $F^{-1}$ exists and is unique. Let $y(t)$ be a WSS zero-mean unit-variance Gaussian random process with an autocorrelation function $\rho(\Delta t)=E\{y(t) y(t+\Delta t)\}$. It is known that $\mu(t)$ can be generated by the nonlinear transformation [13, Ch. 3.1.1]

$$
\mu(t)=g(y(t))
$$

where $g(x)=F^{-1}(\Phi(x))$. In addition, $R_{\mu}(\Delta t)$ and $\rho(\Delta t)$ are related by ([13, eq. (3.7)]

$$
\begin{aligned}
& R_{\mu}(\Delta t)= \int_{-\infty}^{\infty} \int_{-\infty}^{\infty} g\left(y_{1}\right) g\left(y_{2}\right) \frac{1}{2 \pi \sqrt{1-\rho(\Delta t)^{2}}} \\
& \cdot \exp \left[-\frac{y_{1}^{2}+y_{2}^{2}-2 \rho(\Delta t) y_{1} y_{2}}{2\left(1-\rho(\Delta t)^{2}\right)}\right] d y_{1} d y_{2} \\
& \rho(\Delta t) \neq \pm 1 .
\end{aligned}
$$

In special cases of $\rho(\Delta t)= \pm 1$, it is easy to show that

$$
R_{\mu}(\Delta t)= \begin{cases}m, & \rho(\Delta t)=+1 \\ \int_{0}^{1} F^{-1}(x) F^{-1}(1-x) d x, & \rho(\Delta t)=-1 .\end{cases}
$$


Numerical integration of (26a) is made easy by using the transformation $y_{1}=x_{1}$ and $y_{2}=\rho(\Delta t) x_{1}+\left(1-\rho(\Delta t)^{2}\right)^{1 / 2} x_{2}$ followed by another transformation $x_{1}=(2 s)^{1 / 2} \cos \theta$ and $x_{2}=(2 s)^{1 / 2} \sin \theta$. It follows that (26a) can be simplified to

$$
\begin{gathered}
R_{\mu}(\Delta t)=\frac{1}{2 \pi} \int_{0}^{2 \pi} \int_{0}^{\infty} g(\sqrt{2 s} \cos \theta) \cdot g(\rho(\Delta t) \sqrt{2 s} \cos \theta \\
\left.+\sqrt{1-\rho(\Delta t)^{2}} \sqrt{2 s} \sin \theta\right) \cdot e^{-s} d s d \theta \\
\rho(\Delta t) \neq \pm 1 .
\end{gathered}
$$

The inner and outer integrals can be readily computed by the Gauss-Laguerre rule and the Simpson's rule, respectively. Table I lists the computed $R_{\mu}(\Delta t) / R_{\mu}(0)$ values against $\rho(\Delta t)$ for $0.5 \leq m<1$. Note that $R_{\mu}(0)=m$.

\section{APPENDIX C}

PROOF OF (20)

Consider

$$
\tilde{R}_{w}(\Delta t)=\sum_{\Delta n=-\infty}^{\infty} R_{w}\left(\Delta n \cdot T_{s}\right) \delta\left(\Delta t-\Delta n \cdot T_{s}\right)
$$

It follows that $\tilde{R}_{w}(\Delta t)=R_{w}(\Delta t) \sum_{\Delta n=-\infty}^{\infty} \delta\left(\Delta t-\Delta n \cdot T_{s}\right)$, so that

$$
\begin{aligned}
\tilde{S}_{w}(f) & =\int_{-\infty}^{\infty} e^{-j 2 \pi f \Delta t} \tilde{R}_{w}(\Delta t) d \Delta t \\
& =S_{w}(f)^{*} \sum_{\Delta n=-\infty}^{\infty} \frac{1}{T_{s}} \delta\left(f-\frac{\Delta n}{T_{s}}\right)
\end{aligned}
$$

where * denotes convolution. In general, there are many $S_{w}(f)$ 's that yield the same $\tilde{S}_{w}(f)$. Since we are desired to generate $w(t)$ only at equally $T_{s}$-spaced time instants, by the sampling theorem it is only necessary to consider a $S_{w}(f)$ that is bandlimited for $|f| \leq\left(2 T_{s}\right)^{-1}$. In this special case

$$
S_{w}(f)= \begin{cases}T_{s} \times \tilde{S}_{w}(f), & |f| \leq 1 /\left(2 T_{s}\right) \\ 0, & \text { otherwise }\end{cases}
$$

Taking the Fourier transform of (27), and noting that $R_{w}(\Delta n$. $\left.T_{s}\right)=R_{w}^{*}\left(-\Delta n \cdot T_{s}\right)$ and only a finite number of $R_{w}\left(\Delta n \cdot T_{s}\right)$ 's are available, we get (20).

\section{ACKNOWLEDGMENT}

The authors would like to thank the anonymous reviewers for various useful comments and suggestions.

\section{REFERENCES}

[1] C. C. Watterson, J. R. Juroshek, and W. D. Bensema, "Experimental confirmation of an HF channel model," IEEE Trans. Commun., vol. COM-18, pp. 792-803, Dec. 1970.

[2] M. Nakagami, "The $m$-distribution-A general formula of intensity distribution of rapid fading," in Statistical Methods of Radio Wave Propagation, W. C. Hoffman, Ed. Oxford, U.K.: Pergamon, 1960, pp. 3-36.
[3] P. Hoeher, "A statistical discrete-time model for the WSSUS multipath channel," IEEE Trans. Veh. Technol., vol. 41, pp. 461-468, Nov. 1992.

[4] P. M. Crespo and J. Jiménez, "Computer simulation of radio channels using a harmonic decomposition technique," IEEE Trans. Veh. Technol., vol. 44, pp. 414-419, Aug. 1995.

[5] K. W. Yip and T. S. Ng, "Efficient simulation of digital transmission over WSSUS channels," IEEE Trans. Commun., vol. 43, pp. 2907-2913, Dec. 1995.

[6] K. W. Yip and T. S. Ng, "Karhunen-Loève expansion of the WSSUS channel output and its application to efficient simulation," IEEE J. Select. Areas Commun., vol. 15, pp. 640-646, May 1997.

[7] J. F. Mastrangelo, J. J. Lemmon, L. E. Vogler, J. A. Hoffmeyer, L. E. Pratt, and C. J. Behm, "A new wideband high frequency channel simulation system," IEEE Trans. Commun., vol. 45, pp. 26-34, Jan. 1997.

[8] M. Pätzold, U. Killat, F. Laue, and Y. Li, "On the statistical properties of deterministic simulation models for mobile fading channels," IEEE Trans. Veh. Technol., vol. 47, pp. 254-269, Feb. 1998.

[9] H. Kong and E. Shwedyk, "A hidden Markov model (HMM)-based MAP receiver for Nakagami fading channels," in Proc. 1995 IEEE Int. Symp. Information Theory, Whistler, BC, Canada, Sept. 17-22, 1995, p. 210.

[10] W. R. Braun and U. Dersch, "A physical mobile radio channel model," IEEE Trans. Veh. Technol., vol. 40, pp. 472-482, May 1991.

[11] U. Dersch and R. J. Rüegg, "Simulation of the time and frequency selective outdoor mobile radio channel," IEEE Trans. Veh. Technol., vol. 42, pp. 338-344, Aug. 1993.

[12] N. L. Johnson, S. Kotz, and N. Balakrishnan, Continuous Univariate Distributions, 2nd ed. New York: Wiley, 1995, vol. 2.

[13] M. Grigoriu, Applied Non-Gaussian Processes. Englewood Cliffs, NJ: Prentice-Hall, 1995.

[14] H. Cramér, Mathematical Method of Statistics. Princeton, NJ: Princeton Univ. Press, 1946.

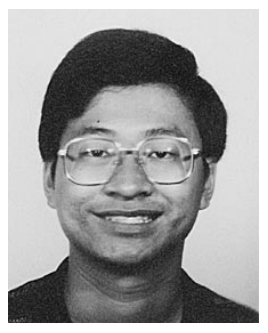

Kun-Wah Yip (M'96) received the B.Eng. (with honors) and Ph.D. degrees in electrical engineering from The University of Bradford, Bradford, UK, in 1991, and The University of Hong Kong in 1995, respectively.

From 1995 to 1998 , he was a Research Associate and then a Postdoctoral Fellow at The University of Hong Kong, where he is currently a Research Assistant Professor. His research interest is on spreadspectrum communications, multicarrier signal transmission, efficient simulation techniques, and theory and techniques for communications over frequency-selective fading channels.

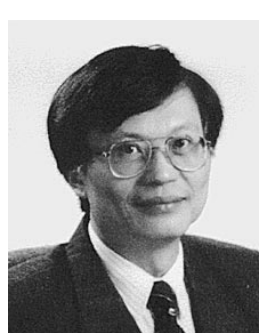

Tung-Sang Ng (S'74-M'78-SM'90) received the B.Sc.(Eng.) degree from the University of Hong Kong in 1972, and the M.Eng.Sc. and Ph.D. degrees from the University of Newcastle, Australia, in 1974 and 1977, respectively, all in electrical engineering.

After graduation, he worked for BHP Steel International and The University of Wollongong, Australia, for 14 years. He returned to Hong Kong in 1991, taking the position of Professor and Chair of Electronic Engineering. He has published over 170 international journal and conference papers. $\mathrm{He}$ is currently a Regional Editor of the International Journal-Engineering Applications of Artificial Intelligence (Pergamon Press). His current research interests include mobile communication systems, spread-spectrum techniques, CDMA, and digital signal processing.

Dr. Ng is a Fellow of the IEE, HKIE, and IEAust. He is also an Executive Committee Member and a Board Member of the IEE Informatics Divisional Board. He was the General Chair of the ISCAS'97and is currently the Vice President for Region 10 of the IEEE CAS Society. He was awarded the Honorary Doctor of Engineering Degree by the University of Newcastle, in August 1997 , for his services to higher education generally and to engineering education specifically. 